\title{
Construction of a high-density linkage map and detection of sex-specific markers in Penaeus japonicus
}

\author{
Yaqun Zhang Equal first author, 1 , Chuantao Zhang ${ }^{\text {Equal first author, } 2}{ }^{2}$, Na Yao ${ }^{1}$, Jingxian Huang ${ }^{2}$, Xiangshan Sun ${ }^{2}$, Bingran Zhao \\ Corresp., 2 , Hengde Li ${ }^{\text {Corresp. } 1}$ \\ ${ }^{1}$ Chinese Academy of Fishery Sciences, Beijing, China \\ 2 Xiaying Enhancement and Experiment Station, Chinese Academy of Fishery Sciences, Weifang, Shandong, China \\ Corresponding Authors: Bingran Zhao, Hengde Li \\ Email address: bran6888@163.com, hengde.li@cafs.ac.cn
}

Penaeus japonicus is one of the most important farmed shrimp species in many countries. Sexual dimorphism is observed in $P$. japonicus, in which females grow faster and larger than males; therefore, a unisexual female culture of $P$. japonicus could improve the efficiency of productivity. However, the genetic mechanisms underlying sex determination in $P$. japonicus are unclear. In this study, we constructed a high-density genetic linkage map of $P$. japonicus using genotyping-by-sequencing (GBS) technology in a full-sib family. The final map was 3,481.98 cM in length and contained 29,757 single nucleotide polymorphisms (SNPs). These SNPs were distributed on 41 sex-averaged linkage groups, with an average inter-marker distance of $0.123 \mathrm{cM}$. One haplotype, harboring five sexspecific SNPs, was detected in linkage group 1 (LG1), and its corresponding confidence interval ranged from 211.840 to $212.592 \mathrm{cM}$. Therefore, this high-density genetic linkage map will be informative for genome assembly and marker-assisted breeding, and the sexlinked SNPs will be helpful for further studies on molecular mechanisms of sex determination and unisexual culture of $P$. japonicus in the future. 


\section{Construction of a high-density linkage map and detection of sex-specific}

\section{2 markers in Penaeus japonicus}

3

4 Yaqun Zhang ${ }^{a \sharp}$, Chuantao Zhang ${ }^{\mathrm{b} \#}$, Na Yao ${ }^{\mathrm{a}}$, Jingxian Huang ${ }^{\mathrm{b}}$, Xiangshan Sun ${ }^{\mathrm{b}}$, Bingran Zhao ${ }^{\mathrm{b}^{*}}$,

5 Hengde $\mathrm{Li}{ }^{*}$

6

7 a Key Laboratory of Aquatic Genomics, Ministry of Agriculture and Rural Affairs, CAFS Key

8 Laboratory of Aquatic Genomics and Beijing Key Laboratory of Fishery Biotechnology, Chinese

9 Academy of Fishery Sciences, Beijing 100141, China.

$10{ }^{\mathrm{b}}$ Xiaying Enhancement and Experiment Station, Chinese Academy of Fishery Sciences, Weifang 11261312 , China.

12

13 *Corresponding authors:

14 Bingran Zhao

15 Tel: +86-536-7582036

16 Email: bran6888@163.com

17 Hengde Li

18 Tel: +86-10-68673905

19 Email: hengde.li@cafs.ac.cn 


\section{Abstract}

22 Penaeus japonicus is one of the most important farmed shrimp species in many countries. Sexual 23 dimorphism is observed in P. japonicus, in which females grow faster and larger than males; 24 therefore, a unisexual female culture of P. japonicus could improve the efficiency of productivity. 25 However, the genetic mechanisms underlying sex determination in P. japonicus are unclear. In 26 this study, we constructed a high-density genetic linkage map of P. japonicus using genotyping27 by-sequencing (GBS) technology in a full-sib family. The final map was 3, $481.98 \mathrm{cM}$ in length 28 and contained 29,757 single nucleotide polymorphisms (SNPs). These SNPs were distributed on 2941 sex-averaged linkage groups, with an average inter-marker distance of $0.123 \mathrm{cM}$. One 30 haplotype, harboring five sex-specific SNPs, was detected in linkage group 1 (LG1), and its corresponding confidence interval ranged from 211.840 to $212.592 \mathrm{cM}$. Therefore, this highdensity genetic linkage map will be informative for genome assembly and marker-assisted breeding, and the sex-linked SNPs will be helpful for further studies on molecular mechanisms of sex determination and unisexual culture of P. japonicus in the future.

35

\section{6}

\section{Introduction}

The kuruma shrimp, Penaeus japonicus, is considered to be one of the most economically important members of the family Penaeidae, and it is distributed along the east coast of South Africa and in Red Sea, Indian Ocean, Korea, Japan, China, Malaysia, Philippines, Indonesia, Fiji Island, and North Australia (Hayashi, 1996). The annual production of $P$. japonicus in China was approximately 50,000 tons from 2013 to 2019 (Ministry of Agriculture and Rural Affairs fishery _. and Fishery Administration., 2013-2019). Sexual dimorphism, in which females grow faster and achieve a larger size than males, occurs in Penaeus shrimps. Therefore, the growth superiority of female penaeid shrimp provides researchers an incentive to investigate the potential of 
45 producing and culturing all-female shrimp populations (Khatkar, 2017). By removing slow46 growing males, it is likely that the culturing of all-female shrimp could increase production and 47 reduce the cost of farming. Therefore, the mechanism of sex determination in Penaeus has long 48 been a question of great interest to researchers.

49 Genotyping-by-sequencing (GBS) is a method used to discover and genotype genome-wide high50 throughput single nucleotide polymorphisms (SNPs) simultaneously, and it functions by reducing genome complexity, relying on restriction enzymes and high-throughput sequencing technology (Wallace and Mitchell, 2017). Construction of genetic maps, which can obtain genomic and genetic variation information based on thousands of SNPs, is an important part of animal and plant molecular breeding and is of great significance for the rapid and scientific identification of molecular markers of target traits (Huang, 2016; You et al., 2020).

Simple sequence repeats (SSRs) are approximately $1 \%$ in most genomes of species and are considered to have no function; however, penaeid shrimp genomes have a high proportion of SSRs (>23\%) (Yuan et al., 2021), which hinders genome assembly. Recently, the genome sequence of the Pacific white shrimp L. vannamei was reported to cover $\sim 1.66 \mathrm{~Gb}$ with 25,596 protein-coding genes and a high proportion of SSRs. Genome sequence assembly provides insights into the genetic underpinnings of specific biological processes and valuable information

62 for promoting crustacean aquaculture (Zhang et al., 2019). In addition to L. vannamei, genome sequencing and draft assembly of two economically important penaeid shrimps, P.japonicus and P. monodon, have also been reported, only at the scaffold level (Yuan et al., 2018). Recently, a chromosome-level assembly of the black tiger shrimp, $P$. monodon, was completed (Uengwetwanit et al., 2021). High-density genetic maps are essential and helpful for genome assembly at higher levels, comparative genomic analysis, and fine mapping of complex traits. To 
68 date, with the rapid development and application of high-throughput sequencing technology, 69 high-density genetic maps of aquatic shrimps, including Litopenaeus vannamei (Peng et al., 2020;

70 Yu et al., 2015; Zhang et al., 2007), Penaeus monodon (Guo et al., 2019; Wilson et al., 2002), 71 and Fenneropenaeus chinensis (Li et al., 2006; Wang et al., 2012), have been completed for 72 reduced-representation genome sequencing. Based on this, the genetic mechanisms underlying 73 sex determination have also been explored. In L. vannamei (Yu et al., 2017), 11 significant SNPs 74 (in high linkage disequilibrium) located on LG42 and 44 involved in sex determination were 75 identified. Sex locus was detected in P. monodon and was speculated to be the same, based on 76 sequence alignments in populations of Mozambique, India, and Hawaii (Guo et al., 2019). In 77 2013, the first genetic map of the kuruma prawn P. japonicus was constructed using AFLP 78 markers (Li et al., 2003), and 217 markers were ordered into 43 linkage groups (1,780 cM) of the 79 paternal map, while 125 markers were ordered into 31 linkage groups $(1,026 \mathrm{cM})$ of the maternal 80 map. In 2016, a higher-resolution genetic linkage map containing 9,289 SNP markers, spanning $813,610.90 \mathrm{cM}$ and ordered into 41 linkage groups, was constructed using RAD technology. 82 Growth-related quantitative trait locus (QTL) has also been identified in P. japonicus (Lu et al., 83 2016). However, there are little published data on the molecular markers of sex determination in $84 \quad$ P.japonicus.

85 To explore the genetic basis of sex determination in $P$. japonicus, a genetic linkage map with 86 higher resolution was constructed using GBS technology, and the sex-specific QTL was 87 identified using chi-square test in this study, our results support the WZ/ZZ sex determination 88 system.

89

90 Materials \& Methods

91 


\section{Sample collection}

93 The full-sib kuruma prawn family that was used for QTL mapping was an F2 population. In 2020,

94 one full-sib family, including F1 parents $(n=2)$ and F2 offspring ( $n=200)$ were randomly collected

95 from the Xiaying Enhancement and Experiment Station, Chinese Academy of Fishery Sciences.

96 Sex was determined by observing their sexual characteristics, the male shrimp has a male

97 appendage on the inner edge of the second appendage, the female shrimp has a seminal vesicle

98 located between the base of the fourth and fifth pairs of feet.One appendage for each prawn was

99 sampled for DNA extraction.

100

101

\section{DNA extraction, library construction, and sequencing}

102 Genomic DNA was extracted from each individual using TIANamp Marine Animals DNA Kit 103 (TIANGEN, Beijing, China) and qualified using gel electrophoresis. DNA concentrations were 104 measured on a Nanodrop and diluted to $50 \mathrm{ng} / \mu \mathrm{L}$. GBS technology was used to construct 105 sequencing libraries (Qi et al., 2018). The DNA concentration of each GBS library was quantified using a Qubit ${ }^{\mathrm{TM}}$ dsDNA HS assay kit. Then libraries were equally pooled and sequenced on an

107 Illumina Nova platform (paired-end 150 bp).

108 Genotyping

109 The raw reads were first split by barcode using the module 'process_radtags' within the Stacks 110 v2.1 (Catchen et al., 2013) (options: -r -renz_1 --adapter_mm 1), then forward reads were filtered 111 using barcode and restriction enzyme sites; they were considered qualified if they simultaneously 112 carried both the barcode and the Pst I restriction site. The restriction sites and all bases at the 3' 113 end with scores less than 20 were removed using the FASTX Toolkit v0.0.14 package 114 (http://hannonlab.cshl.edu/fastx_toolkit/). The clean reads within each sample were clustered 
115 using the 'ustacks' module (options: -m 2 -M 1 -N 1) of the Stacks (Catchen et al., 2013). The

116 representative tags were identified across samples with 'ASustacks', and tag reads were removed

117 if they occurred in less than $50 \%$ of samples or had similarity of $\geq 98 \%$ between samples.

118 Preprocessed reads were aligned to the de novo reference sequences using bowtie2 (v2.3.4.3)

119 (Langmead and Salzberg, 2012) with default parameters, and the genotypes were called using

120 GATK (v3.8-1) (McKenna et al., 2010). To obtain robust results in subsequent analyses, the 121 genotypes were filtered based on three criteria using vcftools (v0.1.13) (Danecek et al., 2011): loci

122 with sequencing depth $<8$, minor allele frequency $(\mathrm{MAF})<0.01$, and call rate $<80 \%$ were 123 removed.

124 Linkage map construction

125 Linkage map was constructed using Lep-MAP3, which can handle large number of markers 126 (Rastas, 2017) and involved several steps as follows: calling parental genotypes using ParentCall2 127 module, followed by filtering markers based on high segregation distortion (options: 128 dataTolerance 0.0001 -MAFLimit 0.05 -missingLimit 0.2) using Filtering2 module. Thereafter, 129 markers were assigned to linkage groups (LGs) by computing all pair-wise likelihood of odds 130 (LOD) scores between markers using SeparateChromosomes2 module, and joining markers with 131 LOD scores higher than the parameter of "usePhysical" as 1, in which LOD score of 12 was used 132 as the threshold. Finally, the markers within each LG were ordered by maximizing the likelihood 133 of the data for alternative orders using OrderMarkers2 module. Joinsignles2All module was not 134 used because only four markers were not assigned to the existing LGs. A linkage map was drawn 135 using the R package 'LinkageMapView' (Ouellette et al., 2018).

136 Sex-specific marker mapping and verification 
137 Missing genotype information was imputed through linkage disequilibrium with 20 closest

138 neighboring markers as previously described by Jiang and Li (2017). The final genotypic data

139 (Table S1) in the analysis were used to calculate the genotype frequency of each SNP, and then a

140 chi-square test was performed to determine the association between the genotypes and the sexes.

141 A genome-wise significance threshold was set as $1.0 \times 10^{-8}$ of $P$-value. The corresponding

142 confidence interval was calculated using the method described by Li (2011). PCR primers (Table

143 1)were designed to detect the sex-specific markers according to the contigs containing the

144 identified markers (Table S2). The PCR reaction conditions were as follows: initial denaturation

145 at $95{ }^{\circ} \mathrm{C}$ for $3 \mathrm{~min} ; 35$ cycles at $95{ }^{\circ} \mathrm{C}$ for $20 \mathrm{~s}$ for denaturation, $60{ }^{\circ} \mathrm{C}$ for $20 \mathrm{~s}$ for annealing, and 72

$146{ }^{\circ} \mathrm{C}$ for $30 \mathrm{~s}$ for extension, followed by a final extension at $72{ }^{\circ} \mathrm{C}$ for $5 \mathrm{~min}$. The amplified products

147 were separated using 1.5\% agarose gel electrophoresis, and the purified products were sequenced.

148 The sex-specific SNP markers were determined manually by the peaks of sanger sequencing of

149 PCR products. Furthermore, more adult shrimps from other group were used to validate the SNP 150 markers (38 females, 51 males).

151

152 Results

153 Genotypes

154 Parents and 200 progenies generated 229.96 million clean reads, comprising approximately 312.77

$155 \mathrm{~Gb}$ of sequencing data, with $49.28 \% \mathrm{GC}$ content (Table S2). A total of 68,457 SNPs were detected 156 and successfully genotyped using GATK pipeline. After quality control, 29,773 high-quality SNPs 157 were obtained.

158 Linkage map

159 Among the 29,773 SNPs, only four SNPs were not mapped to the genetic linkage map in the 160 SeparateChromosomes2 step. In addition, two LGs comprising only three SNPs and nine SNPs, 
161 respectively, which are relatively too short for one chromosome, were excluded from the map. The

162 final map consisted of 41 LGs, harboring 29,757 SNPs. The total map length was 3,481.982 cM,

163 with an average inter-locus distance of $0.123 \mathrm{cM}$. The genetic length of LGs ranged from 54.491

164 (LG29) to $214.101 \mathrm{cM}$ (LG1), with an average inter-locus distance of $0.098 \mathrm{cM}$ (Table 2 and

165 Figure 1). The sex-averaged map information is presented in Table S3. The lengths of the maternal

166 and paternal maps were 3,481.982 and 3,469.499 cM, respectively, and correspondingly, they

167 ranged from 50.584 (LG36) to $214.675 \mathrm{cM}$ (LG1) and from 52.74 (LG37) to $169.327 \mathrm{cM}$ (LG1),

168 with an average inter-locus distance of 0.125 and $0.123 \mathrm{cM}$, respectively (Table S4).

169 Sex QTL and validation of sex associated markers

170 One cluster of five SNPs around position $212.34 \mathrm{cM}$ on LG1 was associated with the sex of $P$.

171 japonicus (Figure 2), in which alleles formed two haplotypes, $\mathrm{H}_{1}$ : GCAGC, $\mathrm{H}_{2}$ : CTCAT, and the

172 genotypes of females and males were $\mathrm{H}_{1} \mathrm{H}_{2}$ and $\mathrm{H}_{1} \mathrm{H}_{1}$, respectively (Figure 3), indicating a WZ/ZZ

173 sex determination system in P. japonicus. The QTL confidence interval ranged from 211.840 to

$174212.592 \mathrm{cM}$ in LG1. The tag sequence containing sex-specific loci was aligned against NCBI $P$.

175 japonicus genome database, "the retrotransposon: Penelope-like element" (NCBI ID:

176 AB612264.1) was hit, then blastx was conducted using the NCBI AB612264.1 sequence against

177 Nr database, and the putative reverse transcriptase (NCBI ID: BAM35674.1) was annotated with

178 an E value of 0 . Primers were designed for the six potential sex-specific markers according to the

179 corresponding contigs, and validated in another population (38 females, 51 males). They were

180 successfully amplified (Figure 3), sequencing results demonstrated that females are all

181 heterozygous and males are all homozygous for these five sex-specific markers, and the

182 specificity of these 5 markers in the discrimination of the population were all $100 \%$. 


\section{Discussion}

184 Sexual dimorphism is observed in P. japonicus, in which females grow faster and larger than 185 males; therefore, a unisexual female culture of $P$. japonicus could improve the efficiency of 186 productivity. Therefore, the sex determination system and the genomic regions associated with sex 187 in P. japonicus needs to be explored. This study identified a QTL region containing 5 markers that 188 explains $100 \%$ of the phenotypic variation in sex. These markers may be beneficial to identify 189 underlying sex-determination genes, and also could be utilized to increase the proportion of 190 females within the industry of kuruma prawn.

191 Lu et al. (2007) reported the genetic map of P. japonicus containing 9,289 SNP markers and 192 spanning 3,610.90 cM with an average marker interval of $0.388 \mathrm{cM}$, and all the SNP markers were 193 grouped into 41 LGs in the maps. In this study, the marker density of the constructed genetic map 194 was three times higher than that reported previously, the final map of $P$. japonicus consisted of 195 29,757 SNPs that also clustered into $41 \mathrm{LGs}$; the average inter-locus distance was $0.123 \mathrm{cM}$; the higher resolution of the genetic map is beneficial to QTL mapping and genomic selection of 197 growth, disease resistance and other complex traits in P. japonicus. $27.14 \%$ scaffolds (4943/18210) of the assembled P. japonicus genome GCA_017312705.1 were mapped using 199 contigs harboring 29,757 SNPs consisting of the final genetic map, indicating that the genetic map could provide a reference for chromosome-level assembly of the $P$. japonicus genome. Notably, karyotype analysis showed that $P$. japonicus has 43 chromosomes (Xiang et al., 1991), which is inconsistent to the 41 LGs obtained in this study. The corresponding number of LGs, which changed with the LOD limit, is shown in Figure S1, the number of LGs with no less than 10 markers tended to be stable at 41 when the LOD limit was between 12 and 21 . What needs to be 
$206 \mathrm{cM}$, which was far below the length of the shortest LG $(57.752 \mathrm{cM})$ in the final genetic map, 207 together with that previous karyotype analysis showed that no extreme short chromosomes existed 208 in P. japonicus, therefore, these two LGs were excluded from the final genetic map. We infer that 209 chromosome rearrangements might occur in P. japonicus, leading to changes in chromosome 210 numbers. Recently, chromosome rearrangements have been detected in the barred knifejaw 211 Oplegnathus fasciatus, and a centric fusion of acrocentric chromosomes $\mathrm{Ch} 8$ and $\mathrm{Ch} 10$ should be 212 responsible for the formation of the $\mathrm{X}_{1} \mathrm{X}_{2} \mathrm{Y}$ system (Xiao et al., 2020). In addition, the difference 213 in chromosomal numbers based on karyotyping and next generation sequencing could be due to 214 the misidentification of chromosome numbers, especially in species with large chromosome 215 numbers such as crustacean species (Waiho et al., 2021).

216 Sex determination and differentiation processes has been among the most interesting topics in 217 aquaculture. Sexual dimorphism is directly related to the economic benefits of some aquatic 218 species, such as the crabs Scylla paramamosain and Eriocheir sinensis, and the females are 219 preferred because of the yolk; the female Chinese tongue soles (Cynoglossus semilaevis) are more 220 preferred owing to their faster growth rate and larger individual size than those of the males. 221 Previous studies have identified sex-related QTLs and genes in fish (Sun et al., 2017; Wei et al., 222 2019), crabs (Waiho et al., 2019), and shrimps, including L. vannamei and P. monodon (Jones et 223 al., 2020; Wang et al., 2020). In L. vannamei, sex-associated markers were identified on LG42.44 224 225 via mapping analysis, and the QTL region supports the ZW-WW chromosomal sex determination system; however, no direct sex determination or differentiation gene could be identified in this 226 research (Jones et al., 2020). Four validated sex-linked SNPs on two sex-linked genes unigene 0020898 and unigenen 0020336 were identified by another research group, and the two 228 genes might participate in sex determination and differentiation processes in L. vannamei (Wang 
229 et al., 2020). One sex locus was located (Guo et al., 2019; Robinson et al., 2014; Staelens et al., 230 2008), supporting that the sex of the black tiger shrimp is determined by a WZ/ZZ chromosomal 231 system. However, studies on sex determination system of $P$. japonicus and sex-related markers are 232 much limited. In this study, a sex-linked significant QTL including five SNPs was detected in LG1 233 of P. japonicus, which were heterozygous in all females but homozygous in all males, the 234 segregation patterns of females:males for these five SNPs are 1:1, completely conforms to 235 Mendelian separation law, also suggesting WZ/ZZ sex determination system in P. japonicus. One 236 reverse transcriptase was annotated by this region. However, it has not been reported to be relevant to sex determination, further studies are needed to identify the genes involved in sex-determining mechanisms in Penaeus species. As sexual dimorphism is observed in P. japonicus in which females grow faster and larger than males, the unisexual female culture could improve the productivity, however, it is difficult to distinguish the sex of $P$. japonicus at their early

241 developmental stages. In this study, sex-specific primers were designed to amplify the region 242 containing sex-related SNPs, and this PCR-based sex identification method were validated to 243 identify the sex of P. japonicus successfully. Therefore, this sex QTL not only offers clues to 244 explore the underlying molecular mechanism of sex determination and differentiation but could 245 also be applied to sex identification and manipulation in the kuruma prawn industry, which leads 246 to the increase of production in $P$. japonicus industry.

247

\section{Conclusions}

249 GBS technology was applied to construct a high-density genetic linkage map for kuruma prawn 250 P. japonicus in this study, this high-quality genetic linkage map will provide a reference for further genome assembly and genomic selection for important economic traits. Our results suggest a

$252 \mathrm{WZ} / \mathrm{ZZ}$ sex determination system in P. japonicus. The identified sex QTL did not only lay a 
253

254

255

256

257

258

259

260

261

262

263

264

265

266

267

268

269

270

271

272

273

274

275

276

277

278

279

280

281

282

283

284

285

286

research foundation for investigating the molecular mechanism of sex determination and

differentiation of $P$. japonicus, but also provides theoretical support for possible unisexual

breeding.

\section{Acknowledgements}

This work was supported by the Central Public-interest Scientific Institution Basal Research

Fund [grant number CAFS: NO. 2021A004]; and Special Scientific Research Funds for Central

Non-profit Institutes [grant number CAFS: 2020TD24]. We would like to thank Editage

(www.editage.cn) for English language editing.

\section{Data statement}

The sequencing data of GBS sequencing have been submitted to the Sequence Read Archive

(SRA) database of NCBI with the accession code PRJNA739820.

\section{References}

Catchen, J., Hohenlohe, P.A., Bassham, S., Amores, A., Cresko, W.A., 2013. Stacks: an analysis tool set for population genomics. Mol. Ecol. 22, 3124-3140.

Danecek, P., Auton, A., Abecasis, G., Albers, C.A., Banks, E., DePristo, M.A., Handsaker, R.E., Lunter, G., Marth, G.T., Sherry, S.T., McVean, G., Durbin, R., 2011. The variant call format and VCFtools. Genomes project analysis, G. Bioinformatics 27, 2156-2158.

Guo, L., Xu, Y.H., Zhang, N., Zhou, F.L., Huang, J.H., Liu, B.S., Jiang, S.G., Zhang, D.C., 2019. A high-density genetic linkage map and QTL mapping for sex in black tiger shrimp (Penaeus monodon). Front. Genet. 10, 326.

Huang, X., 2016. From genetic mapping to molecular breeding: genomics have paved the highway. Mol. Plant 9, 959-960.

Jiang, L., Li, H., 2017. Single locus maintains large variation of sex reversal in half-smooth tongue sole (Cynoglossus semilaevis). G3 (Bethesda) 7, 583-589.

Jones, D.B., Nguyen, H.T., Khatkar, M.S., Simma, D.B., Jerry, D.R., Raadsma, H.W., van der Steen, H., Prochaska, J., Zenger, K.R., 2020. The identification of a major sex QTL in the white-leg shrimp, Litopenaeus vannamei. Aquaculture 529, 735673.

Khatkar, M.S., 2017. Genomic selection in aquaculture breeding programs, Bioinformatics in Aquaculture, in Liu Z (Eds). Bioinformatics in Aquaculture: Principles and Methods. pp. 380-391.

Waiho, K., Shi, X., Fazhan. H., Li, S., Zhang, Y., Zheng, H., Liu, W., Fang, S., Ikhwanuddin, M., 
Ma, H., 2019. High-density genetic linkage maps provide novel insights into ZW/ZZ Sex determination system and growth performance in mud crab (Scylla paramamosain), Front. Genet. 10, 298.

Waiho, K., Fazhan, H., Ikhwanuddin, M., Quinitio, E., Baylon, J., Shu-Chien, A.C., Liew, H.J., Afiqah-Aleng, N., Ma, H., 2021. Chromosomal sex determination system in brachyurans and its potential application in aquaculture, Aquaculture 543, 736990.

Langmead, B., Salzberg, S.L., 2012. Fast gapped-read alignment with Bowtie 2. Nat. Methods 9, 357-359.

Li, H., 2011. A quick method to calculate QTL confidence interval. J. Genet. 90, 355-360.

Li, Y.T., Byrne, K., Miggiano, E., Whan, V., Moore, S., Keys, S., Crocos, P., Preston, N., Lehnert, S., 2003. Genetic mapping of the kuruma prawn Penaeus japonicus using AFLP markers. Aquaculture 219, 143-156.

Li, Z., Li, J., Wang, Q., He, Y., Liu, P., 2006. AFLP-based genetic linkage map of marine shrimp Penaeus (Fenneropenaeus) chinensis. Aquaculture 261, 463-472.

Lu, X., Luan, S., Cao, B., Meng, X., Sui, J., Dai, P., Luo, K., Shi, X., Hao, D., Han, G., Kong, J., 2017. Estimation of genetic parameters and genotype-by-environment interactions related to acute ammonia stress in Pacific white shrimp (Litopenaeus vannamei) juveniles at two different salinity levels. PLOS ONE 12, e0173835.

Lu, X., Luan, S., Hu, L.Y., Mao, Y., Tao, Y., Zhong, S.P., Kong, J., 2016. High-resolution genetic linkage mapping, high-temperature tolerance and growth-related quantitative trait locus (QTL) identification in Marsupenaeus japonicus. Mol. Genet. Genomics 291, 1391-1405.

McKenna, A., Hanna, M., Banks, E., Sivachenko, A., Cibulskis, K., Kernytsky, A., Garimella, K., Altshuler, D., Gabriel, S., Daly, M., DePristo, M.A., 2010. The Genome Analysis Toolkit: a MapReduce framework for analyzing next-generation DNA sequencing data. Genome Res. 20, 1297-1303.

Ministry of Agriculture and Rural Affairs fishery and Fishery Administration., 2013-2019. China Fishery Statistical Yearbook. Bejing: China Agriculture Press.Moss, S.M., Arce, S.M., Moss, D.R., Otoshi, C.A., 2003. Disease Prevention Strategies for Penaeid Shrimp Culture. Oceanic Institute.

Ouellette, L.A., Reid, R.W., Blanchard, S.G., Brouwer, C.R., 2018. LinkageMapView-rendering high-resolution linkage and QTL maps. Bioinformatics 34, 306-307.

Peng, M., Zeng, D., Zhu, W., Chen, X., Yang, C., Liu, Q., Li, Q., Wang, H., Liu, H., Liang, J., Lin, Y., Chen, X., Zhao, Y., 2020. Construction of a high-density genetic map and identification of quantitative trait loci for nitrite tolerance in the Pacific white shrimp (Litopenaeus vannamei). Front. Genet. 11, 571880.

Qi, P., Gimode, D., Saha, D., Schröder, S., Chakraborty, D., Wang, X., Dida, M.M., Malmberg, R.L., Devos, K.M., 2018. UGbS-Flex, a novel bioinformatics pipeline for imputation-free SNP discovery in polyploids without a reference genome: finger millet as a case study. BMC Plant Biol. 18, 117.

Rastas, P., 2017. Lep-MAP3: robust linkage mapping even for low-coverage whole genome sequencing data. Bioinformatics 33, 3726-3732.

Robinson, N.A., Gopikrishna, G., Baranski, M., Katneni, V.K., Shekhar, M.S., Shanmugakarthik, J., Jothivel, S., Gopal, C., Ravichandran, P., Gitterle, T., Ponniah, A.G., 2014. QTL for white spot syndrome virus resistance and the sex-determining locus in the Indian black tiger shrimp (Penaeus monodon). BMC Genomics 15, 731. 
333

334

335

336

337

338

339

340

341

342

343

344

345

346

347

348

349

350

351

352

353

354

355

356

357

358

359

360

361

362

363

364

365

366

367

368

369

370

371

372

373

374

375

376

377

378

Shi, X., Waiho, K., Li, X., Ikhwanuddin, M., Miao, G., Lin, F., Zhang, Y., Li, S., Zheng, H., Liu, W., Aweya, J.J., Azmie, G., Baylon, J.C., Quinitio, E.T., Ma, H., 2018. Female-specific SNP markers provide insights into a WZ/ZZ sex determination system for mud crabs Scylla paramamosain, $S$. tranquebarica and $S$. Serrata with a rapid method for genetic sex identification. BMC Genomics 19, 981.

Staelens, J., Rombaut, D., Vercauteren, I., Argue, B., Benzie, J., Vuylsteke, M., 2008. Highdensity linkage maps and sex-linked markers for the black tiger shrimp (Penaeus monodon). Genetics 179, 917-925.

Sun, C., Niu, Y., Ye, X., Dong, J., Hu, W., Zeng, Q., Chen, Z., Tian, Y., Zhang, J., Lu, M., 2017. Construction of a high-density linkage map and mapping of sex determination and growthrelated loci in the mandarin fish (Siniperca chuatsi). BMC Genomics 18, 446.

Uengwetwanit, T., Pootakham, W., Nookaew, I., Sonthirod, C., Angthong, P., Sittikankaew, K., Rungrassamee, W., Arayamethakorn, S., Wongsurawat, T., Jenjaroenpun, P., Sangsrakru, D., Leelatanawit, R., Khudet, J., Koehorst, J.J., Schaap, P.J., Martins Dos Santos, V., Tangy, F., Karoonuthaisiri, N., 2021. A chromosome-level assembly of the black tiger shrimp (Penaeus monodon) genome facilitates the identification of growth-associated genes. Mol. Ecol. Resour., https://doi.org/10.1111/1755-0998.13357.

Wallace, J.G., Mitchell, S.E., 2017. Genotyping-by-sequencing. Curr. Protoc. Plant Biol. 2, 6477.

Wang, W., Tian, Y., Kong, J., Li, X., Liu, X., Yang, C., 2012. Integration genetic linkage map construction and several potential QTLs mapping of Chinese shrimp (Fenneropenaeus chinensis) based on three types of molecular markers. Genetika 48, 508-521.

Wang, Y., Yu, Y., Li, S., Zhang, X., Xiang, J., Li, F., 2020. Sex-specific transcriptome sequencing of zoea I larvae and identification of sex-linked genes using bulked segregant analysis in pacific white shrimp Litopenaeus vannamei. Mar. Biotechnol. (NY) 22, 423-432.

Wei, J., Chen, Y., Wang, W., 2019. A high-density genetic linkage map and QTL mapping for sex and growth-related traits of large-scale loach (Paramisgurnus dabryanus). Front. Genet. $10,1023$.

Wilson, K., Li, Y., Whan, V., Lehnert, S., Byrne, K., Moore, S., Pongsomboon, S., Tassanakajon, A., Rosenberg, G., Ballment, E., Fayazi, Z., Swan, J., Kenway, M., Benzie, J., 2002. Genetic mapping of the black tiger shrimp Penaeus monodon with amplified fragment length polymorphism. Aquaculture 204, 297-309.

Xiang, J., Zhou, L., Liu, R., Yu, D., 1991. The chromosomes of three shrimp Penaeus penicillatus, P. semisulcatus and P. japonicus. Hai Yang Ke Xue. 4, 74-75 (in Chinese, Abstract in English).

Xiao, Y., Xiao, Z., Ma, D., Zhao, C., Liu, L., Wu, H., Nie, W., Xiao, S., Liu, J., Li, J., HerreraUlloa, A., 2020. Chromosome-level genome reveals the origin of neo-Y chromosome in the male barred knifejaw Oplegnathus fasciatus. iScience 23, 101039.

You, X., Shan, X., Shi, Q., 2020. Research advances in the genomics and applications for molecular breeding of aquaculture animals. Aquaculture 526, 735357.

Yu, Y., Zhang, X., Yuan, J., Li, F., Chen, X., Zhao, Y., Huang, L., Zheng, H., Xiang, J., 2015. Genome survey and high-density genetic map construction provide genomic and genetic resources for the Pacific White shrimp Litopenaeus vannamei. Sci. Rep. 5, 15612.

Yu, Y., Zhang, X., Yuan, J., Wang, Q., Li, S., Huang, H., Li, F., Xiang, J., 2017. Identification of sex-determining loci in pacific white shrimp Litopeneaus vannamei using linkage and association analysis. Mar. Biotechnol. (NY) 19, 277-286.

Peer) reviewing PDF | (2021:06:62589:2:0:NEW 11 Sep 2021) 
Yuan, J., Zhang, X., Liu, C., Yu, Y., Wei, J., Li, F., Xiang, J., 2018. Genomic resources and comparative analyses of two economical penaeid shrimp species, Marsupenaeus japonicus and Penaeus monodon. Mar. Genomics 39, 22-25.

Yuan, J., Zhang, X., Wang, M., Sun, Y., Liu, C., Li, S., Yu, Y., Gao, Y., Liu, F., Zhang, X., Kong, J., Fan, G., Zhang, C., Feng, L., Xiang, J., Li, F., 2021. Simple sequence repeats drive genome plasticity and promote adaptive evolution in penaeid shrimp. Commun. Biol. 4, 186.

Zhang, L., Yang, C., Zhang, Y., Li, L., Zhang, X., Zhang, Q., Xiang, J., 2007. A genetic linkage map of Pacific white shrimp (Litopenaeus vannamei): sex-linked microsatellite markers and high recombination rates. Genetica 131, 37-49.

Zhang, X., Yuan, J., Sun, Y., Li, S., Gao, Y., Yu, Y., Liu, C., Wang, Q., Lv, X., Zhang, X., Ma, K.Y., Wang, X., Lin, W., Wang, L., Zhu, X., Zhang, C., Zhang, J., Jin, S., Yu, K., Kong, J., Xu, P., Chen, J., Zhang, H., Sorgeloos, P., Sagi, A., Alcivar-Warren, A., Liu, Z., Wang, L., Ruan, J., Chu, K.H., Liu, B., Li, F., Xiang, J., 2019. Penaeid shrimp genome provides insights into benthic adaptation and frequent molting. Nat. Commun. 10, 356. 
Figure 1

Figure 1. High-density sex-averaged genetic linkage map of $P$. japonicus . The $X$-axis represents the linkage group, while the $Y$-axis represents the genetic position.

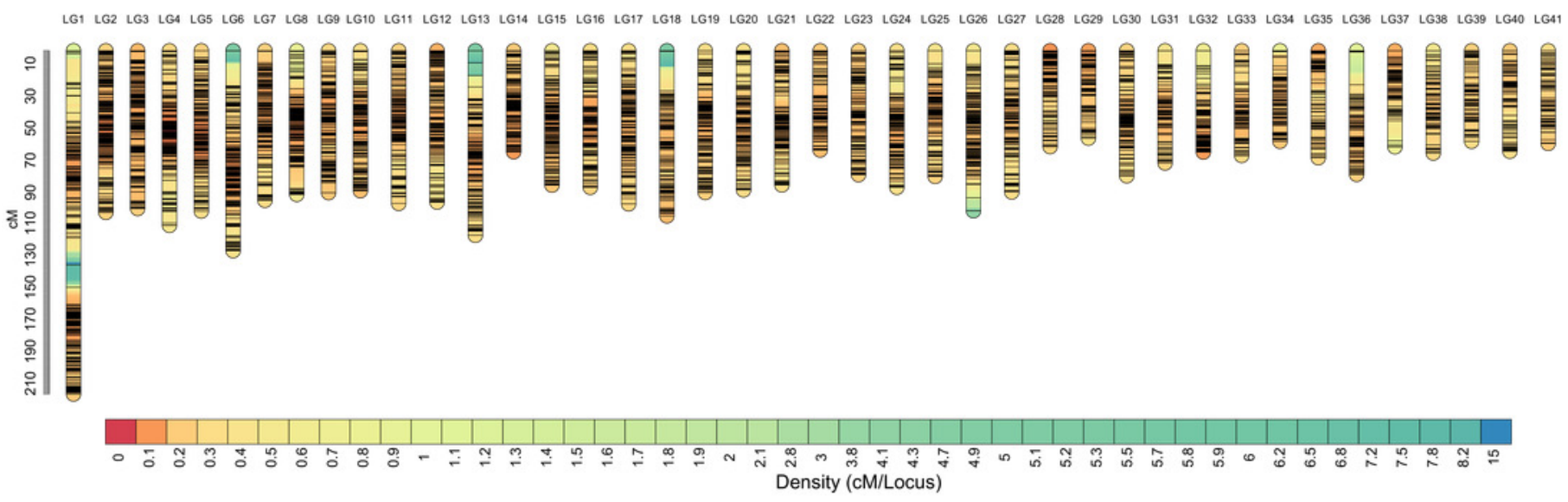


Figure 2

Figure 2. Genome-wide manhattan plot associated to sex in $P$. japonicus.

(A) Manhattan plot of SNPs associated with sex, $x$-axis presents genomic coordinates along chromosome 1-41. The y-axis presents a negative logarithm of P-values. (B) Enlarged plot for LG1, the five sex-related SNPs were arrowed.

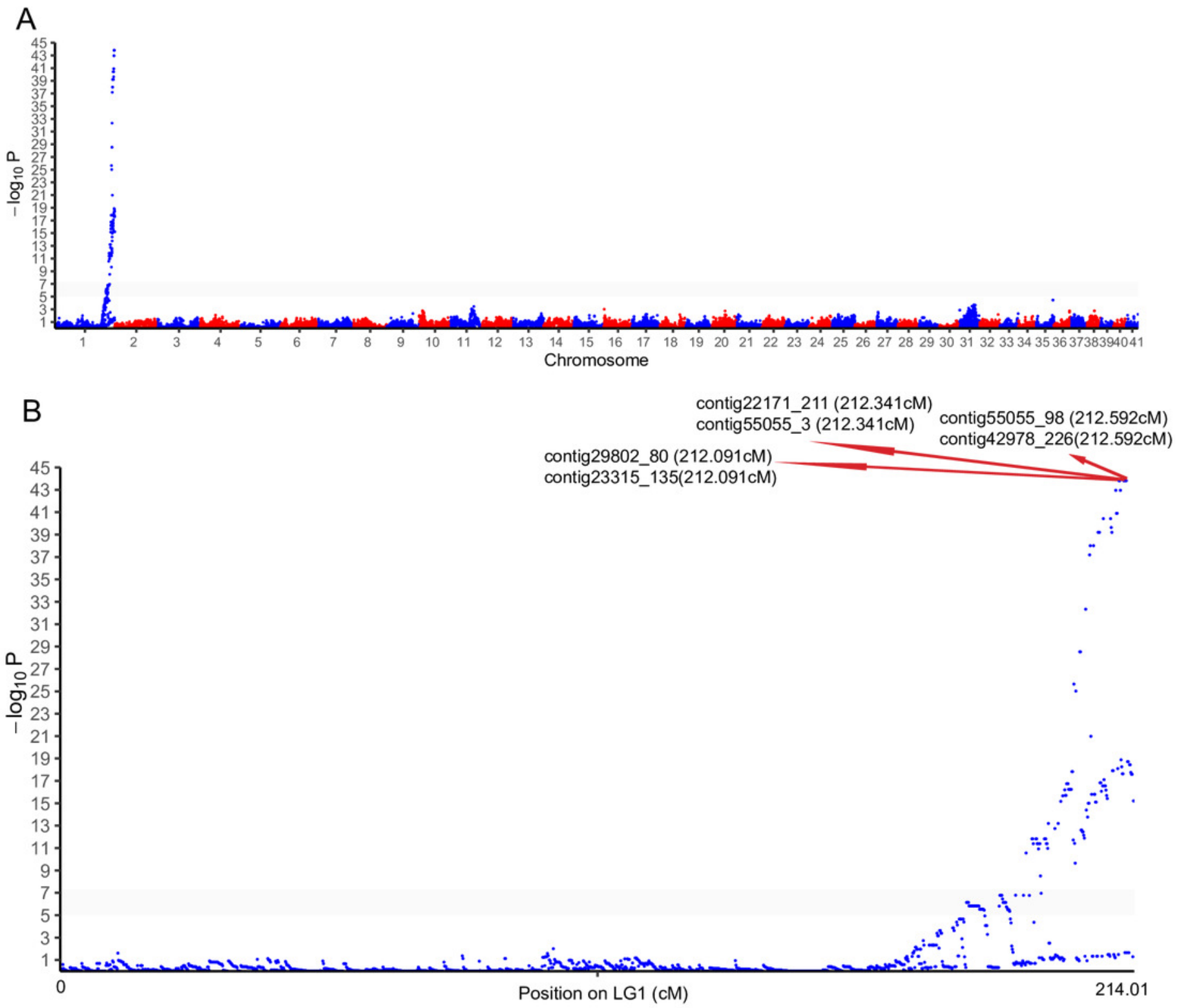




\section{Figure 3}

Figure 3. Verification of the identified sex-related SNPs.

(A) Gel picture of the four contigs containing five sex-related SNPs. (B) Sanger sequencing showed differentiation of SNPs between females and males of $P$. japonicus, contig23315_135, C/G; contig42978_226, C/T; contig55055_3, C/A; contig55055_98, A/G; contig29802_80, C/T. 


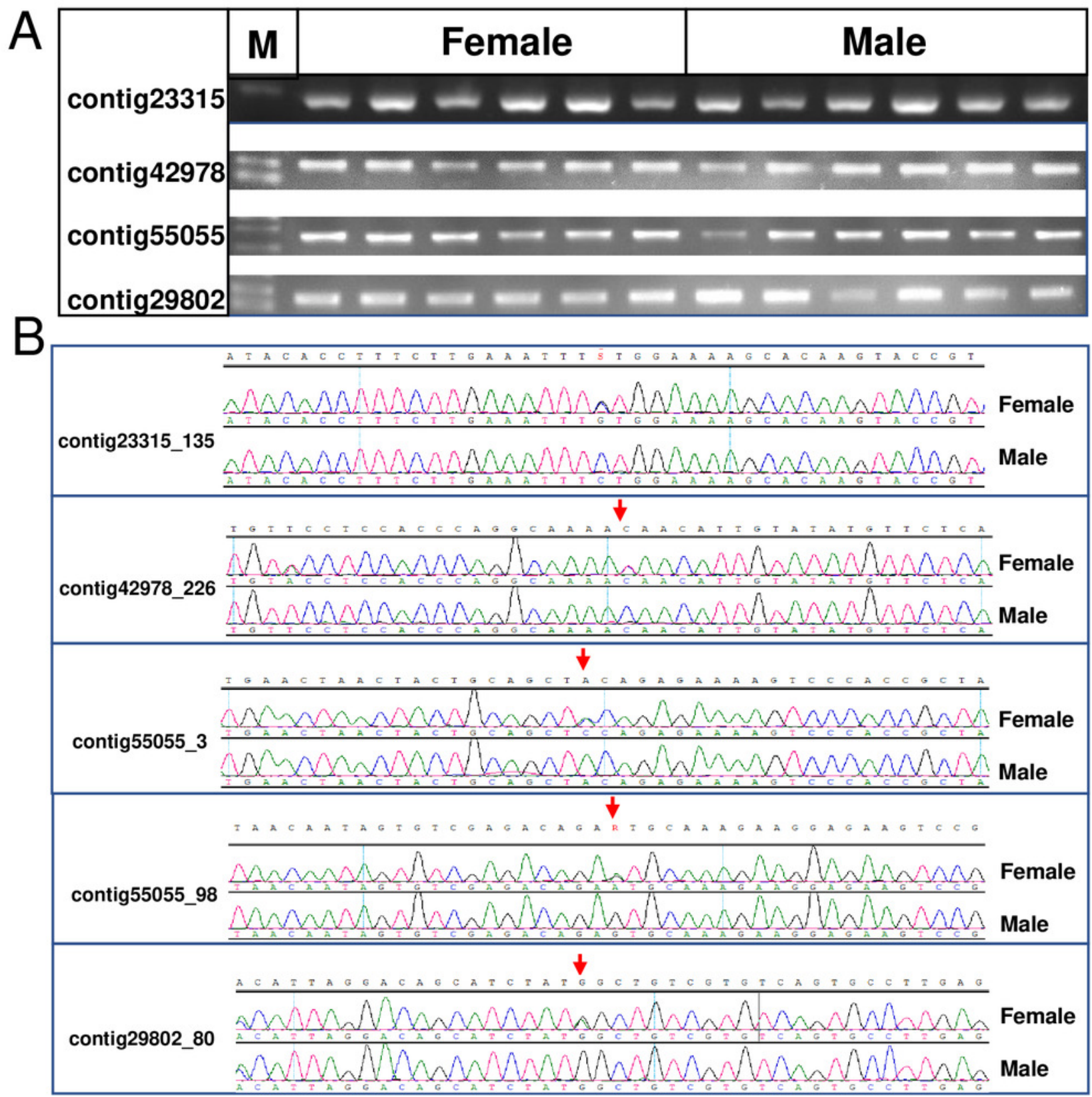




\section{Table $\mathbf{1}$ (on next page)}

Table 1. Sex-specific primer sequences designed according to the genomic sequence containing the identified markers. 
1 Table 1. Sex-specific primer sequences designed according to the genomic sequence 2 containing the identified markers.

\begin{tabular}{ll}
\hline Primer name & Primer sequence (5'-3') \\
\hline contig55055-F & GCGCTGTGCAATATAACAGTCATGG \\
contig55055-R & GTGGAATTATGACAGGTTCTGGACC \\
contig42978-F & TTCGGCATATAGATGGATCC \\
contig42978-R & CACTTCAATGACTCGTTGTG \\
contig29802-F & AACAGATCTCAAGGCACTG \\
contig29802-R & GCAGAACCAATTATGAAGACG \\
contig23315-F & GGATGAGCTGGTACTTCAATCACG \\
\hline contig23315-R & TCAGTGGCGTTTCTCTACCTGTAGG \\
\hline
\end{tabular}

3 
Table 2 (on next page)

Table 2. Summary of the consensus linkage map in Penaeus japonicus. 
1 Table 2. Summary of the consensus linkage map in Penaeus japonicus.

\begin{tabular}{|c|c|c|c|}
\hline $\begin{array}{l}\text { Linkage } \\
\text { group }\end{array}$ & $\begin{array}{c}\text { Number of } \\
\text { markers }\end{array}$ & $\begin{array}{l}\text { Estimated linkage } \\
\text { group length of consensus map }\end{array}$ & $\begin{array}{c}\text { Number of } \\
\text { unique } \\
\text { markers }\end{array}$ \\
\hline 1 & 1640 & 214.101 & 216 \\
\hline 2 & 1182 & 100.837 & 172 \\
\hline 3 & 1171 & 98.44 & 151 \\
\hline 4 & 1105 & 108.945 & 143 \\
\hline 5 & 1096 & 99.991 & 141 \\
\hline 6 & 1044 & 124.737 & 150 \\
\hline 7 & 964 & 93.295 & 132 \\
\hline 8 & 916 & 89.627 & 120 \\
\hline 9 & 886 & 88.463 & 124 \\
\hline 10 & 870 & 87.312 & 125 \\
\hline 11 & 863 & 95.234 & 136 \\
\hline 12 & 852 & 94.383 & 132 \\
\hline 13 & 836 & 115.051 & 126 \\
\hline 14 & 829 & 62.96 & 111 \\
\hline 15 & 825 & 83.777 & 134 \\
\hline 16 & 775 & 85.193 & 112 \\
\hline 17 & 765 & 95.459 & 130 \\
\hline 18 & 714 & 102.954 & 107 \\
\hline 19 & 707 & 88.387 & 124 \\
\hline 20 & 695 & 86.752 & 117 \\
\hline 21 & 681 & 83.739 & 122 \\
\hline 22 & 648 & 61.875 & 78 \\
\hline 23 & 644 & 77.304 & 109 \\
\hline 24 & 643 & 85.181 & 116 \\
\hline 25 & 623 & 78.426 & 97 \\
\hline 26 & 603 & 99.836 & 130 \\
\hline 27 & 583 & 88.12 & 109 \\
\hline 28 & 582 & 59.774 & 93 \\
\hline
\end{tabular}




\begin{tabular}{llll}
29 & 558 & 54.491 & 72 \\
30 & 555 & 77.963 & 107 \\
31 & 553 & 69.978 & 78 \\
32 & 552 & 63.152 & 77 \\
33 & 510 & 65.272 & 69 \\
34 & 488 & 56.542 & 71 \\
35 & 487 & 66.683 & 73 \\
36 & 482 & 77.136 & 81 \\
37 & 431 & 59.904 & 68 \\
38 & 366 & 63.75 & 69 \\
39 & 357 & 56.515 & 68 \\
40 & 352 & 62.691 & 73 \\
41 & 324 & 57.752 & 68 \\
\hline
\end{tabular}

